\title{
Design and validation of a near- infrared fluorescence endoscope for detection of early esophageal malignancy
}

Dale J. Waterhouse

James Joseph

André A. Neves

Massimiliano di Pietro

Kevin M. Brindle

Rebecca C. Fitzgerald

Sarah E. Bohndiek 


\title{
Design and validation of a near-infrared fluorescence endoscope for detection of early esophageal malignancy
}

\author{
Dale J. Waterhouse, ${ }^{a, b}$ James Joseph, ${ }^{a, b}$ André A. Neves, ${ }^{b}$ Massimiliano di Pietro, ${ }^{c}$ Kevin M. Brindle, ${ }^{b, d}$ \\ Rebecca C. Fitzgerald, ${ }^{\mathrm{c}}$ and Sarah E. Bohndiek ${ }^{\mathrm{a}, \mathrm{b}, *}$ \\ aUniversity of Cambridge, Department of Physics, JJ Thomson Avenue, Cambridge CB3 OHE, United Kingdom \\ bUniversity of Cambridge, Cancer Research UK Cambridge Institute, Li Ka Shing Centre, Cambridge CB2 ORE, United Kingdom \\ CUniversity of Cambridge, MRC Cancer Unit, Hutchison/MRC Research Centre, Cambridge CB2 OXZ, United Kingdom \\ dUniversity of Cambridge, Department of Biochemistry, Sanger Building, Cambridge CB2 1GA, United Kingdom
}

\begin{abstract}
Barrett's esophagus is a known precursor lesion to esophageal adenocarcinoma. In these patients, early detection of premalignant disease, known as dysplasia, allows curative minimally invasive endoscopic therapy, but is confounded by a lack of contrast in white light endoscopy. Imaging fluorescently labeled lectins applied topically to the tissue has the potential to more accurately delineate dysplasia, but tissue autofluorescence limits both sensitivity and contrast when operating in the visible region. To overcome this challenge, we synthesized near-infrared (NIR) fluorescent wheat germ agglutinin (WGA-IR800CW) and constructed a clinically translatable bimodal NIR and white light endoscope. Images of NIR and white light with a field of view of 63 deg and an image resolution of $182 \mu \mathrm{m}$ are coregistered and the honeycomb artifact arising from the fiber bundle is removed. A minimum detectable concentration of $110 \mathrm{nM}$ was determined using a dilution series of WGAIR800CW. We demonstrated ex vivo that this system can distinguish between gastric and squamous tissue types in mouse stomachs $(p=0.0005)$ and accurately detect WGA-IR800CW fluorescence in human esophageal resections (compared with a gold standard imaging system, $r_{s}>0.90$ ). Based on these findings, future work will optimize the bimodal endoscopic system for clinical trials in Barrett's surveillance. @ 2016 Society of Photo-Optical Instrumentation Engineers (SPIE) [DOI: 10.1117/1.JBO.21.8.084001]
\end{abstract}

Keywords: Flexible endoscope; fluorescence; Barrett's esophagus; clinical translation; near-infrared; dysplasia.

Paper 160204SSPRR received Apr. 1, 2016; accepted for publication Jul. 13, 2016; published online Aug. 4, 2016.

\section{Introduction}

Barrett's esophagus is an acquired condition that predisposes patients to esophageal adenocarcinoma (EAC), an aggressive cancer with 5-year survival rates as low as $20 \%$. $^{1}$ Progression to cancer occurs through intermediate stages of low-grade dysplasia (LGD) and high-grade dysplasia (HGD). ${ }^{2}$ While the cancer risk in Barrett's patients is typically less than $0.5 \%$ per year, the risk of progression may be up to $10 \%$ in the LGD that emerges within regions of Barrett's. ${ }^{3,4}$ Early resection or radiofrequency ablation of these dysplastic regions can, however, be curative, ${ }^{5,6}$ which has motivated routine clinical surveillance of Barrett's patients. ${ }^{7}$

Current clinical surveillance uses a combination of white light endoscopy (WLE) with targeted and random biopsies. Random biopsies are taken in each quadrant every $2-\mathrm{cm}$ along the length of the Barrett's segment according to the Seattle protocol. ${ }^{8}$ These random biopsies are time consuming, labor intensive, costly, and subject to high sampling error as a result of the patchy distribution of dysplastic regions. ${ }^{9}$ To reduce sampling error, targeted biopsies are guided by WLE, but poor contrast between dysplastic and Barrett's tissues results in a sensitivity as low as $40 \%$ for dysplasia. ${ }^{10,11}$ To overcome this limitation, advanced wide-field surveillance techniques with improved detection of dysplastic regions and thus more accurate targeting of biopsies have been developed. These include: narrow band imaging; ${ }^{12-17}$ autofluorescence imaging, ${ }^{18-23}$ chromoendoscopy; ${ }^{24-27}$ and optical coherence tomography. ${ }^{28-30}$ Despite these recent advances, none has yet been recommended for use in routine clinical practice due to lack of robust evidence on the improved detection of dysplasia by their targeting of biopsies. $^{7,9}$

Molecular imaging enables the visualization of complex biochemical processes involved in both normal physiology and disease states. The use of molecular imaging has the potential to achieve both high sensitivity and specificity for identification of dysplasia. ${ }^{31}$ Previous efforts in the development of molecular imaging contrast agents for this application have been limited by long clearance times of probes after intravenous administration, requiring at least $24 \mathrm{~h}$ between injection and endoscopy. ${ }^{32,33}$ Encouragingly, labels that can be topically applied during the endoscopic procedure have begun to emerge. A fluorescently labeled peptide that binds specifically to HGD and adenocarcinoma has recently been shown by Sturm et al. ${ }^{34}$ to delineate these pathologies in vivo in patients; however, the sensitivity to LGD has yet to be established. A follow-up study by Joshi et al. ${ }^{35}$ using a wide-field fluorescence imaging endoscope noted some important limitations, including: the challenges in visualizing the FITC fluorophore due to strong tissue autofluorescence; and the resulting low values of target-to-background ratio from diagnostic fluorescence features. 
We have previously demonstrated that cell-surface glycans are altered in the progression from Barrett's esophagus to adenocarcinoma. The fluorescently labeled lectin wheat germ agglutinin (WGA) showed specific changes in binding patterns between HGD and LGD tissue and nondysplastic (ND) Barrett's esophagus. ${ }^{36}$ The low-molecular weight fluorescent WGA showed potential for more accurate delineation of dysplasia than WLE and importantly for clinical translation, could be applied topically using a spray catheter and was easily displaced by washing with an excess of glucosamine. ${ }^{36}$ Progression of this and other promising molecular imaging agents into clinical trials, however, has encountered technological limitations. Existing endoscopy devices for detecting fluorescence emissions are optimized for detection of tissue autofluorescence in the visible wavelength range, meaning that the sensitivity, contrast, and dynamic range for detection of exogenously applied contrast agents is limited by the high endogenous background signal present within the images.

To avoid the autofluorescent background, imaging can be performed using near-infrared (NIR) fluorophores in the range $\sim 600$ to $900 \mathrm{~nm}$, where the tissue is relatively devoid of endogenous fluorescence. ${ }^{37}$ This has motivated several groups to develop flexible endoscopic devices for NIR fluorescence imaging in the GI tract, since none are commercially available at present. ${ }^{32,38-40}$ Pilot studies of such devices have been performed ${ }^{40,41}$ and several clinical trials are in progress (NCT01972373, NCT02113202, NCT02129933, and NCT02710838). Still, data supporting the use of flexible NIR fluorescence endoscopes in clinical trials for wide-field surveillance of Barrett's esophagus are lacking.

The overall goal of our work is to translate wide-field NIR fluorescence molecular imaging to improve the detection sensitivity and specificity for both low- and high-grade dysplastic lesions in Barrett's esophagus. Toward this goal, we have labeled WGA with a NIR dye (IR800CW) to create WGAIR800CW and developed a clinically translatable bimodal endoscope to detect both NIR fluorescence for molecular imaging as well as traditional white light reflectance. Our solution is based on an existing clinically approved accessory channel endoscope to ease the translational pathway. Here, we present the design and development of our bimodal endoscopic system, the technical characterization process undertaken along with preliminary biological validation and discuss the next steps required to achieve routine clinical application.

\section{Materials and Methods}

All chemicals were purchased from Sigma-Aldrich unless otherwise stated.

\subsection{Fluorescent Lectin Synthesis}

IRDye800CW NHS Ester was obtained from LI-COR Bioscience. Conjugation of the dye with wheat germ agglutinin (WGA, L9640) was performed according to the methods of Sato et al. $^{42}$ to produce WGA-IR800CW.

\subsection{Endoscope Design}

A schematic of the endoscopic system is shown in Fig. 1. The system is based around an imaging fiber-optic bundle consisting of 10,000 individual fibers (PolyDiagnost). This is threaded inside a disposable catheter that protects the distal tip of the imaging fiber bundle from direct patient contact with a glass cover plane (PolyDiagnost). The catheter also contains a light guide for illumination and a $1.2 \mathrm{~mm}$ working channel. The combined imaging fiber bundle and catheter is known as the PolyScope flexible endoscope. The PolyScope may be used independently or introduced through the accessory channel of another endoscope.

Designing our device around the PolyScope system offers several advantages. First, the lack of direct patient contact removes the need for sterilization of the imaging fiber between procedures, prolonging the lifetime of this expensive component of the system. Second, the system carries a CE mark for endoscopic retrograde cholangio-pancreatography, which will facilitate safety approval for use in the esophagus. Third, the catheter has a maximum diameter of $3.0 \mathrm{~mm}$ allowing it to be threaded into a $3.7 \mathrm{~mm}$ working channel of a commercial endoscope for direct comparison to gold standard white light reflectance imaging performed at the tip of the endoscope.

Illumination is provided by a broadband halogen light source (OSL2B2, Thorlabs) clipped with a $750 \mathrm{~nm}$ short pass excitation filter (FESH0750, Thorlabs). This is directed onto the sample through the light guide embedded inside the PolyScope catheter. Light collected by the imaging fiber is relayed through an infinity corrected 20x objective lens (421350-9970-000, Zeiss) and split into two channels with a long pass dichroic filter (DMLP650L, Thorlabs). Light from 400 to $633 \mathrm{~nm}$ is relayed by an achromatic doublet (ACA254-100-A, Thorlabs) onto a

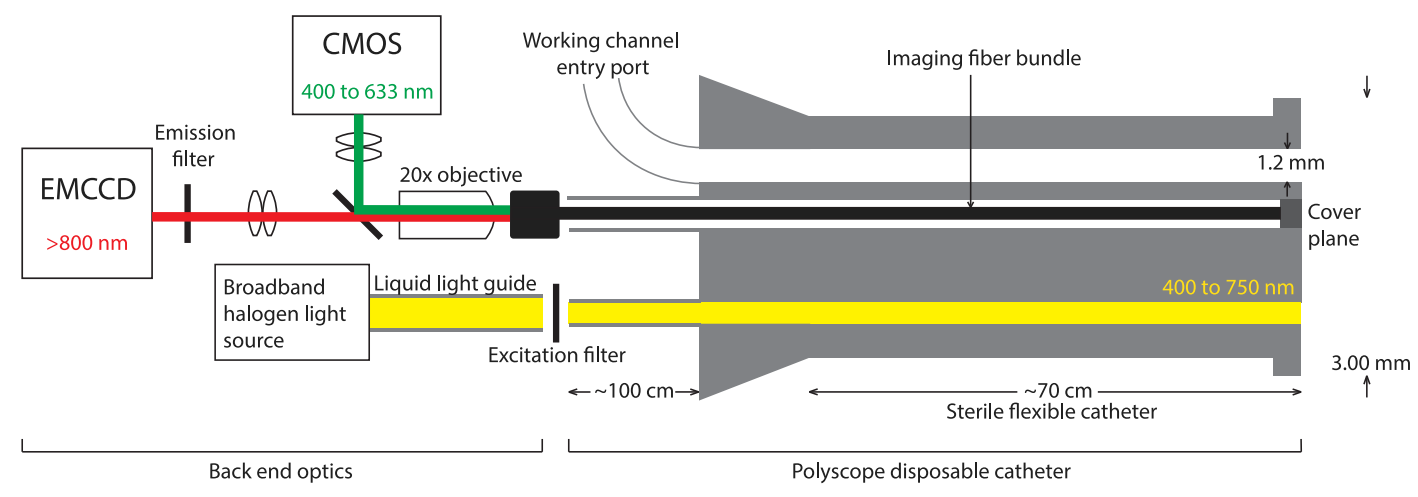

Fig. 1 Schematic of the PolyScope-based bimodal endoscope hardware. The PolyScope disposable catheter can be introduced through the accessory channel of a standard endoscope and protects the imaging fiber bundle from contact with the patient. Light collected by the fiber is split into white light $(400$ to $633 \mathrm{~nm})$ and near-infrared $(>800 \mathrm{~nm})$ channels for imaging. 
grayscale, complementary metal-oxide-semiconductor sensor (CMOS; Grasshopper3 GS3-U3-41C6M-C, PointGrey) while light $>685 \mathrm{~nm}$ is relayed by an achromatic doublet (ACA254-100-B, Thorlabs) onto an electron multiplying charge coupled device (EMCCD; ProEM+ eXcelon $512 \times 512$, Princeton) through an $800 \mathrm{~nm}$ long pass emission filter (FELH0800, Thorlabs), allowing simultaneous WL reflectance and NIR fluorescence imaging.

The system is kept compact through the use of broadband mirrors (BB1-E02 and BB1-E03, Thorlabs) to bend the light path. The optics are securely housed inside a light-tight enclosure and mounted on an optical breadboard (MB4545/M, Thorlabs) that is fixed to a stainless steel trolley (FW2901-3, Freeway Medical) with a footprint of $512 \mathrm{~mm} \times 480 \mathrm{~mm}$. This allows the system to be easily and safely transported around a crowded endoscopy suite.

\subsection{Image Acquisition and Corrections}

A LabView (National Instruments) visual interface is used to control the cameras and acquire images. The white light images are saved as 8 bit $2048 \times 2048$ tiff files and the NIR images are saved as 16 bit $512 \times 512$ tiff files. Data analysis was carried out using MATLAB ${ }^{\circledR}$ (MathWorks).

An overall schematic of the image corrections performed can be seen in Fig. 2. Since the imaging fiber bundle is composed of 10,000 individual fibers, a honeycomb structure appears in the images. To remove this, we first acquire a bright field calibration image from an externally illuminated diffusely reflecting surface (paper). Next, we identify the locations of each individual fiber within these bright field images using an algorithm adapted from Elter et al. ${ }^{43}$ The procedure is as follows:

1. Acquire a bright field calibration image $I$.

2. Define candidate fiber centers $c_{i}\left(x_{i}, y_{i}\right)$ as image points $I\left(x_{i}, y_{i}\right)$ that are at a maximum with respect to image points at one fiber radius separation $r$ :

$$
I\left(x_{i}, y_{i}\right)>I\left(x_{i} \pm r, y_{i} \pm r\right) .
$$

3. For each candidate fiber center $c_{i}\left(x_{i}, y_{i}\right)$, calculate an average difference in brightness

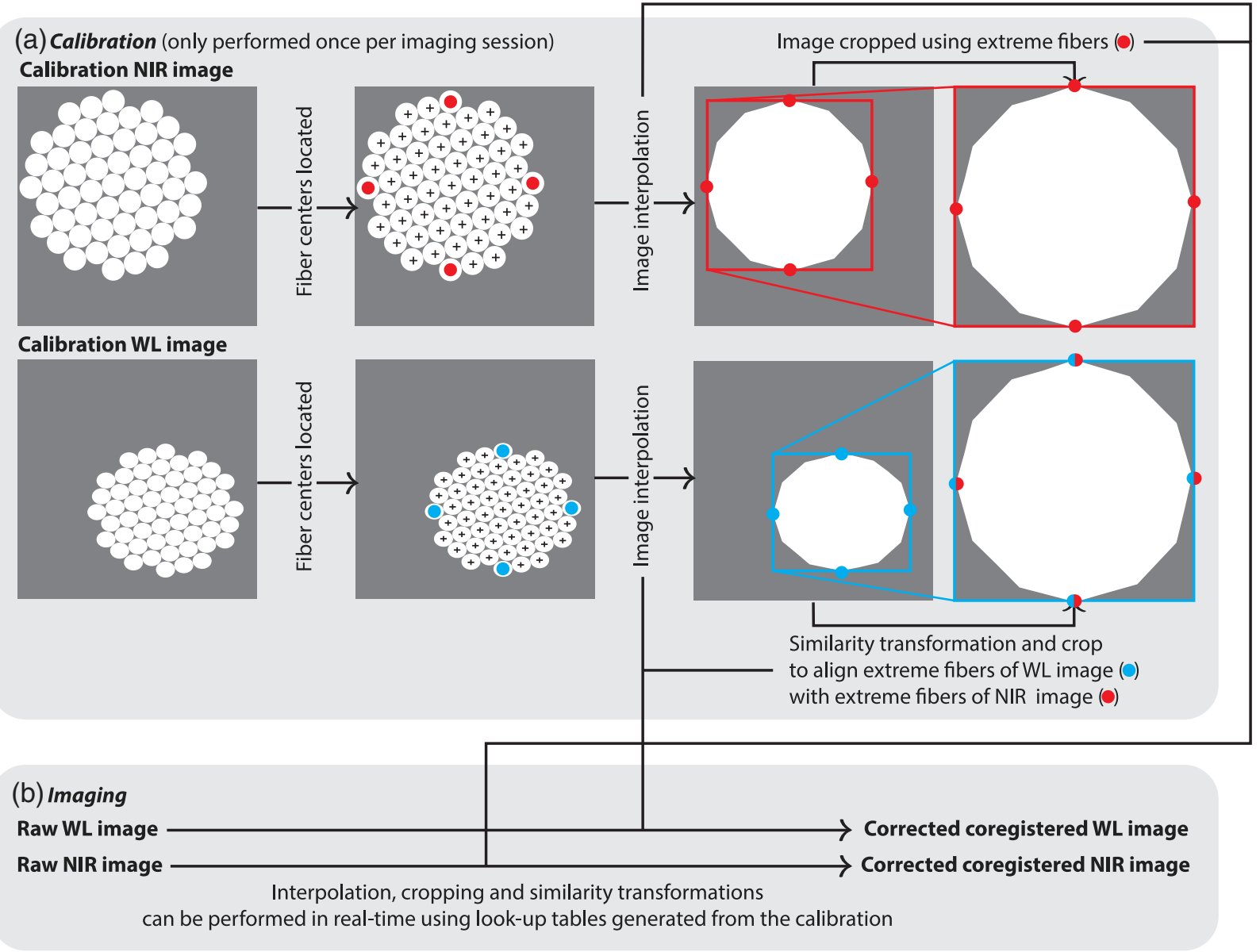

Fig. 2 Schematic of the image correction process. (a) Bright field images contain a honeycomb structure due to the individual fibers in the imaging fiber bundle. Between channels, the images are offset and have different scales in $x$ and $y$. The honeycomb correction algorithm is used to locate the centers of individual fibers (+). The most extreme fiber centers in $x$ and $y$ are also identified (circles). The honeycomb structure is removed by interpolating between image points at fiber center positions. The images are coregistered by using the extreme fiber centers as the bounding points of the image for each channel. (b) After the initial calibration, we can define a set of lookup tables that can be used to directly convert raw images into corrected coregistered images without further calculation. 


$$
D_{i}=\frac{1}{8} \sum\left[I\left(x_{i}, y_{i}\right)-I\left(x_{i} \pm r, y_{i} \pm r\right)\right],
$$

between the candidate pixel and all of its neighbors.

4. Order candidate fiber centers in descending order of $D_{i}$.

5. Starting from the highest ranked candidate (largest $D_{i}$ ), sequentially place each candidate fiber center $c_{i}\left(x_{i}, y_{i}\right)$ onto a center map if and only if the candidate is a minimum distance of one fiber diameter from all centers $m_{j}\left(x_{j}, y_{j}\right)$ already in the map:

$$
\sqrt{\left(x_{i}-x_{j}\right)^{2}+\left(y_{i}-y_{j}\right)^{2}}>2 r .
$$

6. Fiber centers are added for as long as this criterion is satisfied until all candidates have been added to the map or rejected.

Following identification of fiber centers, we reconstruct an image free of honeycomb artifact by calculating a bilinear interpolation of pixel values at fiber centers. Once this procedure is complete, we can define a lookup table that can be used to directly convert raw images into interpolated images without further calculation. This must be updated only if the system is realigned, which allows us to perform the correction in real time. The white light and NIR images can be coregistered using the fiber locations by selecting the four fibers located at the extreme $x$ and $y$ positions. The images are cropped at these locations and a similarity transformation is generated to successfully transform the white light image in $x$ and $y$ such that the extreme fibers are coregistered with the equivalent extreme fibers in the NIR image.

\subsection{Optical Characterization}

\subsubsection{Field of view}

To enable wide-field surveillance of the whole esophagus it is important that the endoscope has sufficient imaging performance across a wide field of view (FOV). In order to measure the FOV we captured 3 images of $1 \mathrm{~mm}$ graph paper at four different working distances (WD): 5.7, 8.7, 11.7, and $16.7 \mathrm{~mm}$ (error $\pm 0.3 \mathrm{~mm}$ ). The resulting images will show a barrel distortion defined by

$r_{u}=A r_{d}\left(1+k r_{d}^{2}\right)$

where $r_{u}$ is the radial distance from the center of the ground truth image to a given vertex $i$ in $\mathrm{mm}, r_{d}$ is the radial distance from the center of the distorted image to the same vertex $i$ in pixels, $k$ is a constant that describes the magnitude of the distortion, and $A$ is a constant used to convert between units of pixels and $\mathrm{mm}$. For each of the 12 images acquired (four working distances $\times$ three replicates) we identified the position of the center of the fiber bundle and plotted the radial distance to each vertex in the graph paper, $r_{d}$ (in pixels), against the true distance, $r_{u}$ (in $\mathrm{mm}$ ), which is known from the markings on the graph paper.

\subsubsection{Power}

To establish a maximum power for our system, we measured the maximum broadband power of a clinical endoscope (Olympus Evis Lucera CVL-260SL Xenon light source with an Olympus
Gastroscope GIF-FQ260Z) and of our endoscope in normal operating conditions, at the same working distance, using a thermal power meter (A-02-D12-BBF-USB, LaserPoint).

\subsubsection{Resolution}

The resolution of the system is fundamentally limited by the fiber bundle structure. To determine the limiting resolution, we took images of a 1951 USAF resolution test target (\#53714 , Edmund Optics) at 4 working distances using external illumination from a broadband halogen light source (OSL2B2, Thorlabs). Background subtraction was performed using an averaged dark image of 10 frames acquired at the end of the experiment. White light images were analyzed since the test target is printed on white reflective photo paper intended for visible imaging. Since both imaging channels are able to resolve the individual fibers of the fiber bundle, the limiting resolution is determined by the fiber bundle properties rather than the properties of the cameras, and so the resolution measured using the white light images is applicable to both imaging channels.

\subsubsection{Sensitivity}

For in vivo application it is important that we are able to detect WGA-IR800CW at low concentrations. The reasons for this are threefold. First, since binding of WGA-IR800CW decreases with the progression of the disease, we must be capable of detecting it at low concentrations to avoid false positives. Second, although WGA occurs naturally in food $^{36}$ and IR800CW can be produced under cGMP conditions, minimizing the amount of any exogenous agent sprayed inside the esophagus is desirable for clinical translation. Third, we would like to minimize nonspecific binding effects that may become most problematic at high concentrations where they reduce our contrast. Therefore, determining the sensitivity of the system is a crucial aspect of the characterization process.

In order to characterize sensitivity, we prepared a two-fold dilution series of WGA-IR800CW in phosphate buffered saline (PBS) and pipetted $30 \mu \mathrm{L}$ of each solution into a well plate ( $\mu$-Slide 18 Well-Flat, ibidi $\mathrm{GmbH}$ ), which had been spraypainted matte black to avoid specular reflections. We then captured images of the dye at 5 different working distances $(5,10$, 12,16 , and $20 \mathrm{~mm}$ ) representative of the range that would be used in vivo. The NIR images were captured using an EM gain of 20 and an exposure time of $200 \mathrm{~ms}$. The images were corrected and coregistered as described in Sec. 2.3. $50 \times 50$ pixel regions of interest (ROIs) were drawn on the white light images inside (signal) and outside (background) of the well. These ROIs were then applied to the coregistered NIR images for analysis in MATLAB ${ }^{\circledR}$. The signal-to-noise ratio (SNR) was calculated using

$\mathrm{SNR}=\frac{S-B}{\sigma}$,

where $S$ is the mean of a Gaussian fitted to the pixel intensity distribution in the signal ROI, $B$ is the mean of a Gaussian fitted to the pixel intensity distribution in the background ROI, and $\sigma$ is the standard deviation of a Gaussian fitted to the pixel intensity distribution in the background ROI.

We also characterized the relationship between the SNR and electron multiplying (EM) gain of the NIR sensor by capturing images of $30 \mu \mathrm{L}$ of $3100 \mathrm{nM}$ WGA-IR800CW with different 
levels of gain at working distances of 5, 9.5, and $12 \mathrm{~mm}$. For an EMCCD we would expect

$\mathrm{SNR} \propto\left(\text { constant }+\frac{1}{G^{2}}\right)^{-\frac{1}{2}}$,

with $G$ the EM gain of the sensor. ${ }^{44}$

\subsection{Biological Validation Studies in Ex Vivo Tissue}

\subsubsection{Imaging of mouse stomachs}

To demonstrate the feasibility of imaging WGA-IR800CW on a tissue background we stained excised mouse stomachs with the fluorescently labeled lectin and acquired imaging data using our endoscope. All animal procedures were conducted in accordance with project and personal licenses issued under the United Kingdom Animals (Scientific Procedures) Act, 1986. Surplus mice from breeding were obtained post mortem $(n=6)$.

Mouse stomachs were prepared by opening and pinning the excised stomachs on parafilm covered cork. The stomachs were washed with PBS to remove contents before the first round of imaging. This washing consisted of tilting the stomachs and pouring the PBS such that it ran from left to right over the stomachs to avoid cross contamination between the tissue types. Data acquisition was then performed using $\mathrm{EM}$ gain $=20$ and $\mathrm{WD}=7 \pm 1 \mathrm{~mm}$. After the baseline imaging, $100 \mu \mathrm{L}$ of $29 \mu \mathrm{g} / \mathrm{mL}(780 \mathrm{nM})$ WGA-IR800CW was evenly pipetted onto the face of the stomachs and left to incubate for $10 \mathrm{~min}$. The stomachs were then imaged four more times: immediately after incubation; after a wash with $5 \mathrm{~mL}$ of PBS; after a further wash of $15 \mathrm{~mL}$ of PBS; and after a final wash of $45 \mathrm{~mL}$ PBS. Each round of imaging captured three images: squamous tissue; gastric tissue; and parafilm covered cork to serve as a background control. For each image, an ROI was drawn in the center of the image and the mean signal calculated by fitting a Gaussian to the pixel intensity distribution.

In order to confirm the differential binding of WGA to squamous and gastric tissue types rather than tissue geometry, we repeated the experiment using an additional wash with $33 \mathrm{ml}$ of $1 \mathrm{mM} \mathrm{N}$-acetyl-D-glucosamine (Sigma-Aldrich) in PBS, which has been shown previously to compete with WGA for sialic acid binding. ${ }^{36}$ The washing was performed in the same manner as described above. Excess glucosamine that did not run off was left to incubate for $5 \mathrm{~min}$ before a final wash of $50 \mathrm{~mL}$ of PBS.

\subsubsection{Imaging of human resection specimens}

In order to validate our system in human tissue we performed a pilot study assessing five endoscopic mucosal resection (EMR) specimens from a patient undergoing endoscopic therapy for Barrett's-related intramucosal EAC. The study was approved by the Cambridgeshire 2 Research Ethics Committee (09/ H0308/118).

Resections were first washed with PBS to remove superficial debris and excess mucus then stained with $10 \mu \mathrm{g} / \mathrm{mL}(268 \mathrm{nM})$ WGA-IR800CW using a small spray bottle to mimic the spray catheter application that would be used in an endoscopic practice. Tissues were left to incubate for $10 \mathrm{~min}$ at room temperature then washed with $15 \mathrm{~mL}$ of cold PBS to remove unbound fluorescent probe. The EMRs were imaged with a Fluobeam ${ }^{\circledR}$ 800 intraoperative wide-field fluorescence imaging system
(Fluooptics, France), taken as the gold standard for imaging of NIR fluorescence, and then with our system.

To fit the entire EMRs ( $\sim 2.5 \mathrm{~cm}$ diameter) into a single field of view, we captured images at a working distance of $\sim 2 \mathrm{~cm}$ using our endoscope, resulting in a decrease in illumination power and resulting SNR. To counter this, we increased our exposure time to $2 \mathrm{~s}$, allowing us to achieve adequate SNR.

In order to compare our endoscope images with the gold standard Fluobeam ${ }^{\circledR}$ images, we performed a simple coregistration of the images. We first manually drew ROIs around the EMR in the Fluobeam ${ }^{\circledR}$ image and the white light endoscope image (which is already coregistered with the NIR endoscope image as described in Sec. 2.3). Using these ROIs, we generated binary masks, which were then coregistered using the "imregtform" function in MATLAB ${ }^{\circledR}$. This generated a similarity transformation that was applied to the raw Fluobeam ${ }^{\circledR}$ image to coregister it with the endoscope images. Finally, using both the endoscope mask and the coregistered Fluobeam ${ }^{\circledR}$ mask, the coregistered images were masked so that only image points inside both of the initial ROIs would be compared in the final coregistered images. This process is summarized in Fig. 3.

After NIR imaging, EMR specimens were fixed in formalin and embedded in paraffin, according to standard histopathological procedures at Cambridge University Hospitals Human Research Tissue Bank. The EMR paraffin block was cut at intervals of $2 \mathrm{~mm}$ and sections mounted onto glass slides. Slides were H\&E stained and scanned using an Axio Scan.Z1 and imported into Zen 2 lite software (both Carl Zeiss Microscopy, Jena). The EMR sections were scored by the study pathologist every $1 \mathrm{~mm}$ for pathological grade according to the Vienna classification, with the help of the ZEN software graphical grid. This allowed construction of a histology grid, which was superimposed manually onto FluoBeam ${ }^{\circledR}$ fluorescence images using methodology established previously. ${ }^{45}$ To facilitate histopathological correlation, the normal oesophageal squamous epithelium and oesophageal gastric/intestinal metaplasia (ND Barrett's) were grouped together as "ND" and any grade of neoplasia, including indefinite for dysplasia (ID), LGD and HGD, and intramucosal cancer, were grouped together as "dysplastic." To illustrate the relationship between the NIR fluorescence data and underlying pathology, we used EMR B (see Sec. 3.2.2) as there were similarly sized areas of ND and dysplastic tissue within this sample.

\section{Results}

\subsection{Optical Characterization}

\subsubsection{Field of view}

We first assessed the FOV of the system. An example image displaying barrel distortion can be seen in Fig. 4(a). The distortion constant $k$ and the constant $A$ were determined by fitting Eq. 4 to the data shown in Fig. 4(b) $\left(R^{2}=0.9949-0.9974\right)$. The values of $k$ and $A$ were then used to determine the FOV $\left(=2 r_{u}\right)$ based on the diameter of the images in pixels $\left(=2 r_{d}\right)$. Combining these data for the four working distances, we determined the angle of the FOV to be $63 \pm 1$ deg [Fig. 4(c)], which compares favorably to the manufacturer specified angle of $70 \mathrm{deg}$.

\subsubsection{Power}

To establish a safe maximum power for our endoscope, we compared its maximum broadband power to that of a clinical 


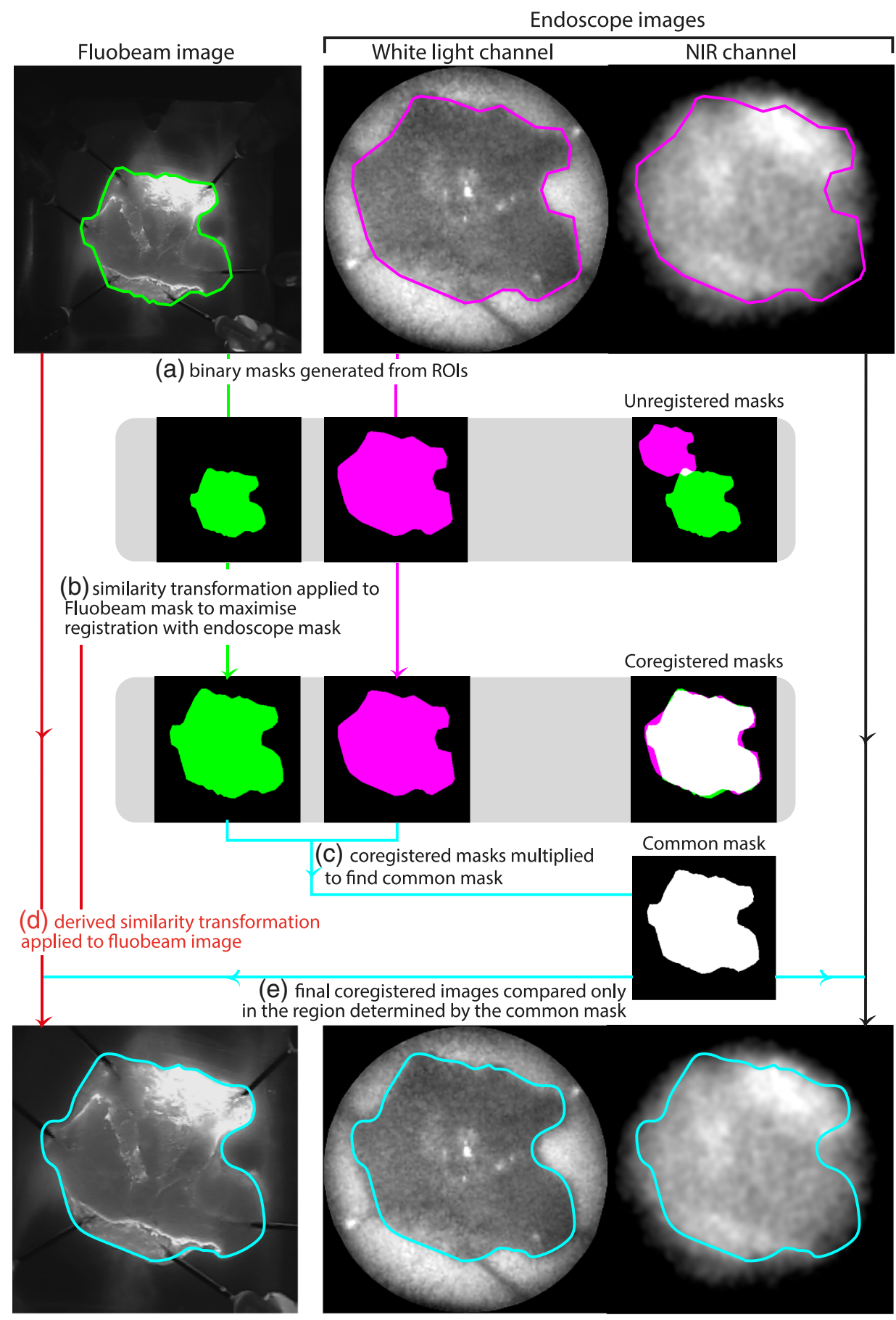

Fig. 3 Coregistration of Fluobeam and endoscope images for validation of ex vivo specimen imaging. (a). ROIs drawn around the EMRs in the Fluobeam image and white light endoscope image are turned into binary masks. Initially these are unregistered. (b) We similarity transform the Fluobeam mask in order to maximize overlap with the endoscope mask. (c) The resulting Fluobeam mask and the endoscope mask are multiplied together to find the common mask. (d) The similarity transform found by coregistering the binary masks is now applied to the Fluobeam image to coregister it with the endoscope image. (e) The coregistered images can be compared in the region defined by the common mask, which represents image points that were found inside both of the original unregistered ROIs.

endoscope. The maximum power from the clinical endoscope in white light reflectance mode was measured to be $19 \pm 1 \mathrm{~mW}$ at a working distance of $1.0 \pm 0.1 \mathrm{~cm}$ while the maximum power from our system was measured to be $3.9 \pm 0.2 \mathrm{~mW}$ at a working distance of $1.0 \pm 0.1 \mathrm{~cm}$.

\subsubsection{Resolution}

Resolution was determined by taking images of a USAF test target. At least four replicate data points for each line pair element were captured (centered within the FOV) for each of 


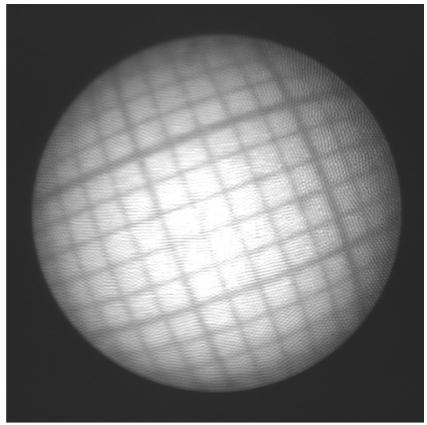

(a)

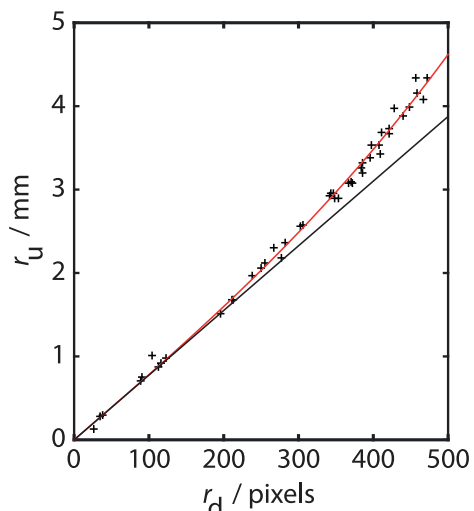

(b)

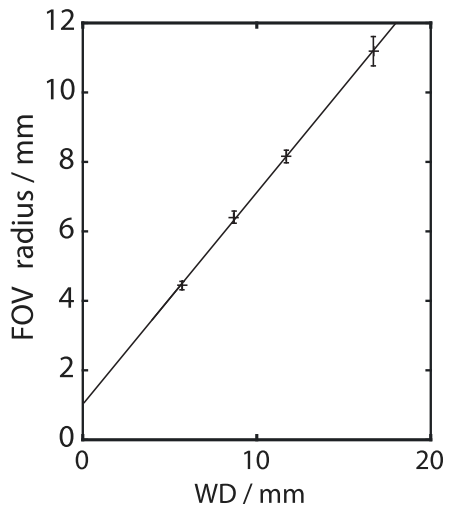

(c)

Fig. 4 Optical characterization of the endoscopy field of view (FOV) and barrel distortion. (a) An endoscopic image of $1 \mathrm{~mm}$ square graph paper shows barrel distortion. From such an image we can measure $r_{u}$ and $r_{d}$ (as defined in Sec. 2.4.1) for several vertices on the paper. (b) An example of the fit to Eq. 4 for images taken at a working distance (WD) of $5.7 \mathrm{~mm}\left(R^{2}=0.9954\right)$. The straight line shows the case for no barrel distortion. The fit was used to extract the constant $A$ and the distortion parameter $k$. The values of $A$ and $k$ can be used with Eq. 4 to determine the FOV $\left(=2 r_{u}\right)$ based on the diameter of the images in pixels $\left(=2 r_{d}\right)$. (c) Determined FOV for four WDs $\left(R^{2}=0.9996\right)$. Error bars represent the standard error of the FOV radius derived from the standard deviations of the fit parameters $A$ and $k$. From the fitted line we calculate the angular FOV to be $63 \pm 1$ deg.

four working distances, 5, 10, 15, and $20 \mathrm{~mm}$. The Michelson contrast was calculated for each element (before and after honeycomb artifact correction) and the results plotted against half the spatial frequency of the element, which is equal to the reciprocal of the width of a single line element (Fig. 5).

Using a model of the modulation threshold, ${ }^{46}$ which has been shown to reproduce the results of several studies, we determined $1 \%$ to be the minimum contrast required for detection of the pattern by an observer viewing the images in normal operating conditions. By finding the intersect of this threshold with linear fits applied in the low frequency region, we determined the resolution of our system to be $182 \pm 2 \mu \mathrm{m}, 210 \pm 30 \mu \mathrm{m}$, $230 \pm 50 \mu \mathrm{m}$, and $290 \pm 60 \mu \mathrm{m}$ at working distances of 5,

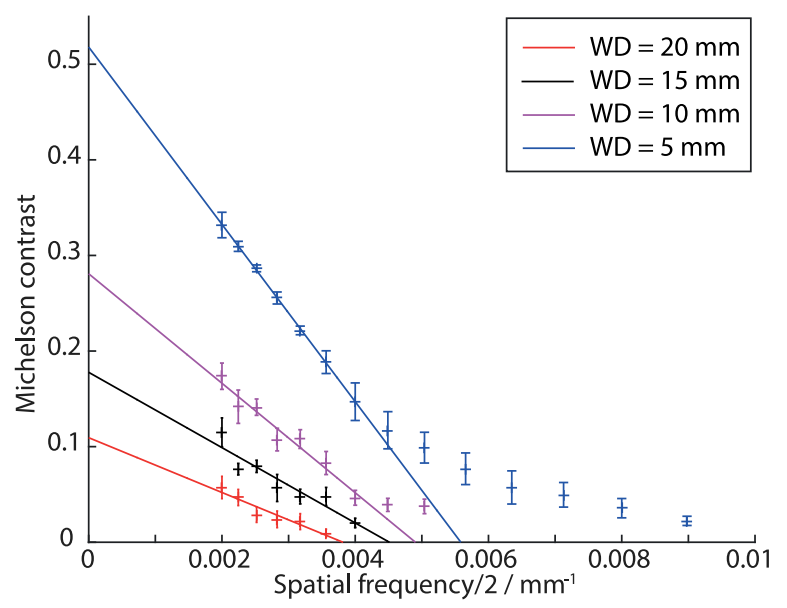

Fig. 5 Extraction of imaging resolution using the Michelson contrast. Resolution was characterized by imaging a USAF chart at four different working distances. The resolution was determined as the point where a linear fit applied in the low-frequency region drops below $1 \%$ Michelson contrast, which was determined to be the minimum contrast required for detection of the pattern by an observer viewing the images in normal operating conditions. $R^{2}=0.9995,0.9567$, 0.8818 , and 0.9138 for working distances of $5,10,15$, and $20 \mathrm{~mm}$, respectively.
10,15 , and $20 \mathrm{~mm}$, respectively. These values are not significantly altered if we do not perform honeycomb correction, being $181 \pm 3 \mu \mathrm{m}, 200 \pm 20 \mu \mathrm{m}, 220 \pm 30 \mu \mathrm{m}$, and $280 \pm 80 \mu \mathrm{m}$, respectively.

\subsubsection{Sensitivity}

Finally, we established the system sensitivity by investigating the dependence of SNR on concentration; a SNR of 3 was used to determine the minimum detectable concentration at each working distance. Example images from the white light and NIR channels are shown in Fig. 6(a) while Fig. 6(b) shows the relationship between SNR and concentration at working distances of 5, 10, and $12 \mathrm{~mm}$. At working distances beyond $16 \mathrm{~mm}$, the dye was not detectable at any concentration $\leq 3100 \mathrm{nM}$. A linear fit is made to these curves for SNR $>1$ since below this we expect only noise in our data. For a working distance of $5 \mathrm{~mm}$, the nonlinear region at high concentration, which is possibly due to saturation, was excluded from the linear fit. The minimum detectable concentrations assessed from Fig. 6(b) are $110 \pm 60 \mathrm{nM}$ and $430 \pm 170 \mathrm{nM}$ for working distances of 5 and $10 \mathrm{~mm}$, respectively. Without the honeycomb correction outlined in Sec. 2.3 the minimum detectable concentrations are $170 \pm 40 \mathrm{nM}$ and $810 \pm 520 \mathrm{nM}$ for working distances of 5 and $10 \mathrm{~mm}$, respectively, suggesting removal of the honeycomb artifact grants us an improvement in SNR.

We also assessed the influence of working distance and EMCCD gain on these data. The relationship between sensitivity and working distance arises due to the dependence on the irradiance of the excitation light. This was investigated by capturing images of $30 \mu \mathrm{L}$ of $3100 \mathrm{nM}$ WGA-IR800CW at eight working distances and plotting SNR against working distance [Fig. 6(c)]. The fit suggests that the SNR falls off as working distance to the power of $-1.91 \pm 0.08$, consistent with the inverse square law expected for an illumination cone.

Figure 6(d) shows the fit of our data to Eq. (6) to assess the relationship between SNR and EMCCD gain. According to our data, using a gain of $\sim 20$ provides optimal SNR while preserving the lifetime of our EMCCD. 
(a)

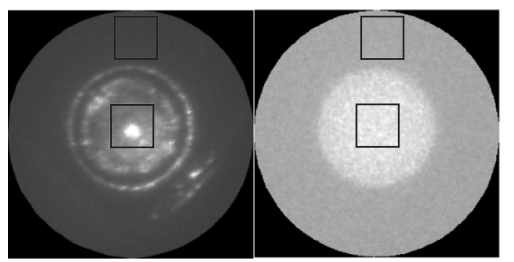

(b)

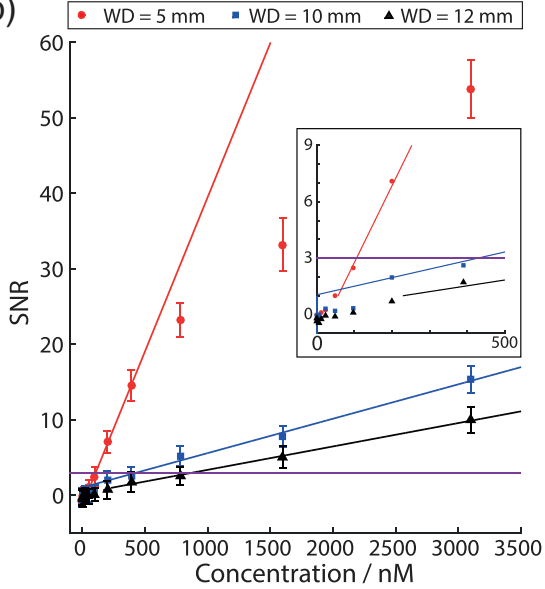

(c)

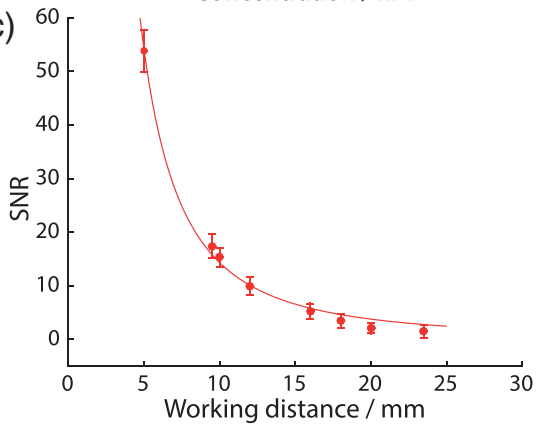

(d) ${ }_{80} \cdot \mathrm{WD}=5 \mathrm{~mm} \quad \mathrm{WD}=9.5 \mathrm{~mm} \triangle \mathrm{WD}=18 \mathrm{~mm}$

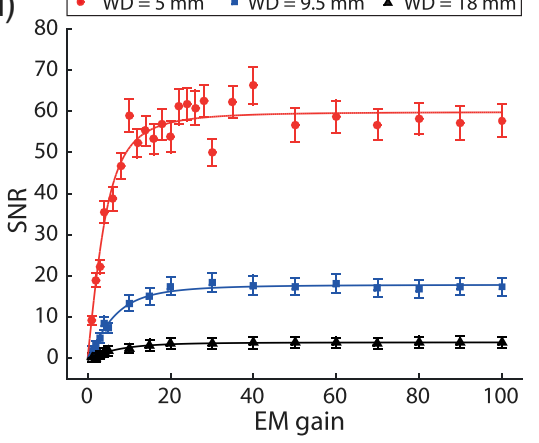

Fig. 6 Endoscope sensitivity for detection of WGA-IR800. (a) Coregistered images of white light (left) and NIR fluorescence (right) with example ROIs illustrated. (b) The signal to noise ratio (SNR) increases with the concentration of WGA-IR800. Linear fits were applied giving $R^{2}=0.9987,0.9936$, and 0.9974 for working distances of 5,10 , and $12 \mathrm{~mm}$, respectively. The horizontal line shows the detection limit defined as $\mathrm{SNR}=3$. Inset shows the region at low concentration. Error bars have been removed for clarity. (c) SNR of $3100 \mathrm{nM}$ WGA-IR800 follows an inverse power law with the working distance: $R^{2}=0.9952$. (d) SNR eventually reaches a plateau with increasing EM gain applied to the EMCCD for images taken of $3100 \mathrm{nM}$ WGA-IR800. The lines show the fit according to Eq. 6. with $R^{2}=0.9358,0.9819$, and 0.9727 for working distances of 5 , 9.5, and $18 \mathrm{~mm}$, respectively. (Exposure time $=200 \mathrm{~ms}$ and $\mathrm{EM}$ Gain $=20$ unless otherwise stated. Error bars represent the standard error of the SNR derived from the standard deviation of pixel values in the signal $\mathrm{ROI}$ ). (a)

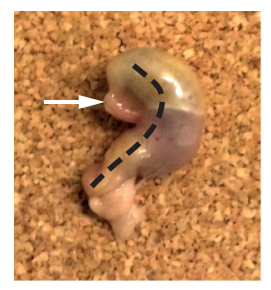

(c)

$\rightarrow$ Squamous tissue

- Gastric tissue

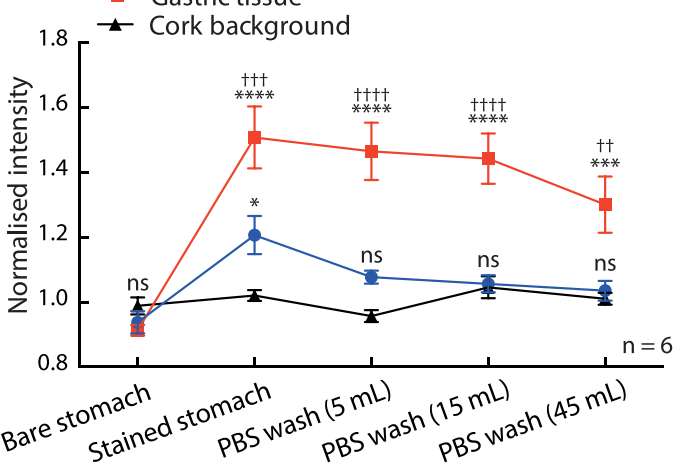

(d)

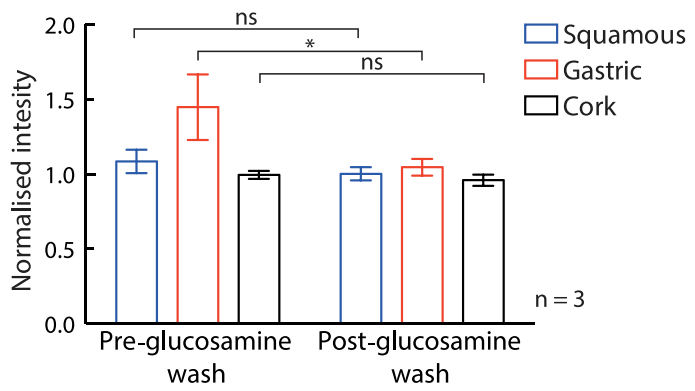

Fig. 7 Fluorescent WGA-IR800 binding to excised mouse stomach. (a), (b) Photographs of the tissue specimens. The arrow in (a) shows the location of the esophagus. The dotted line shows the approximate location of the cut that was made to open the stomach and expose the inner wall as shown in (b). The arrow in (b) shows the direction of washing. In both images we can see the limiting ridge separating the upper nonglandular fore stomach, which has squamous tissue at the exposed surface, and the lower glandular stomach, which has simple columnar epithelium (gastric type) tissue at the exposed surface, which provide us with a model of the corresponding tissue types found in the human esophagus in healthy (squamous) and diseased columnar-lined esophagus or Barrett's, respectively. For each stomach three images were taken: one of the center of the squamous tissue (s); one of the center of the gastric region (g); and one of cork as a background (k). (c) The stomachs were stained with WGA-IR800 and washed with PBS. At each time point, images of each tissue type and cork were taken and the mean intensity in a central ROI of each was calculated. The intensity was normalized to the average background cork level for each mouse. The mean of $n=6$ mice is plotted with error bars representing the standard error in the mean. Statistical testing was carried out using 2 way ANOVA. (ns = no significant difference versus cork; ${ }^{*} p<0.05$ versus cork; ${ }^{* \star *} p<0.001$ versus cork; ${ }^{* * * *} p<0.0001$ versus cork; $\dagger \dagger p<0.01$ versus squamous; $\dagger \dagger \dagger p<0.001$ versus squamous; $\dagger+\dagger \dagger p<0.001$ versus squamous). (d) Following a further wash with glucosamine the bound WGA is removed and the gastric tissue fluorescence intensity returns to the level of the background. Statistical testing was carried out using 2 way ANOVA. (ns $=$ no significant difference; ${ }^{*} p<0.05$ ). 


\subsection{Biological Validation Studies in Ex Vivo Tissue}

\subsubsection{Imaging of mouse stomachs}

To demonstrate the feasibility of imaging WGA-IR800CW on a tissue background we stained excised mouse stomachs with the dye and acquired data using our endoscope. The upper nonglandular fore stomach, which has squamous tissue at the exposed surface, and the lower glandular stomach, which has simple columnar epithelium (gastric type) tissue at the exposed surface, provide us with a model of the corresponding tissue types found in the human esophagus in healthy (squamous) and diseased columnar-lined esophagus or Barrett's, respectively. The different regions of tissue within the stomach are indicated in Figs. 7(a) and 7(b).
Gastric tissue is clearly distinguishable (2 way ANOVA, $p=0.0005)$ from the squamous tissue using our system [Fig. 7(c)], even following extensive washing with PBS, suggesting that the WGA-IR800CW binds preferentially to this mouse tissue. The results shown in Fig. 7(d) show a significant reduction of the fluorescence to background level ( 2 way ANOVA, $p=0.0472$ ) following the glucosamine wash, confirming our results are due to the differential binding of WGA to different tissue types.

\subsubsection{Imaging of human resection specimens}

In a first step towards clinical translation, we performed a pilot study on endoscopic mucosal resections (EMRs) collected from a patient with Barrett's esophagus. The coregistered Fluobeam ${ }^{\circledR}$

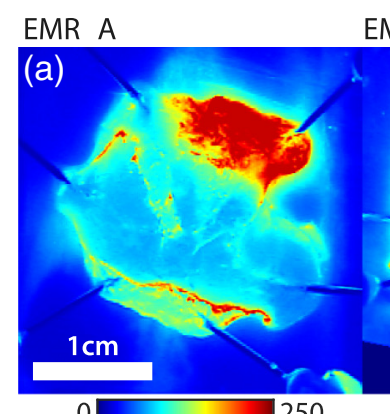

EMR B EMR C

EMR D
EMR E
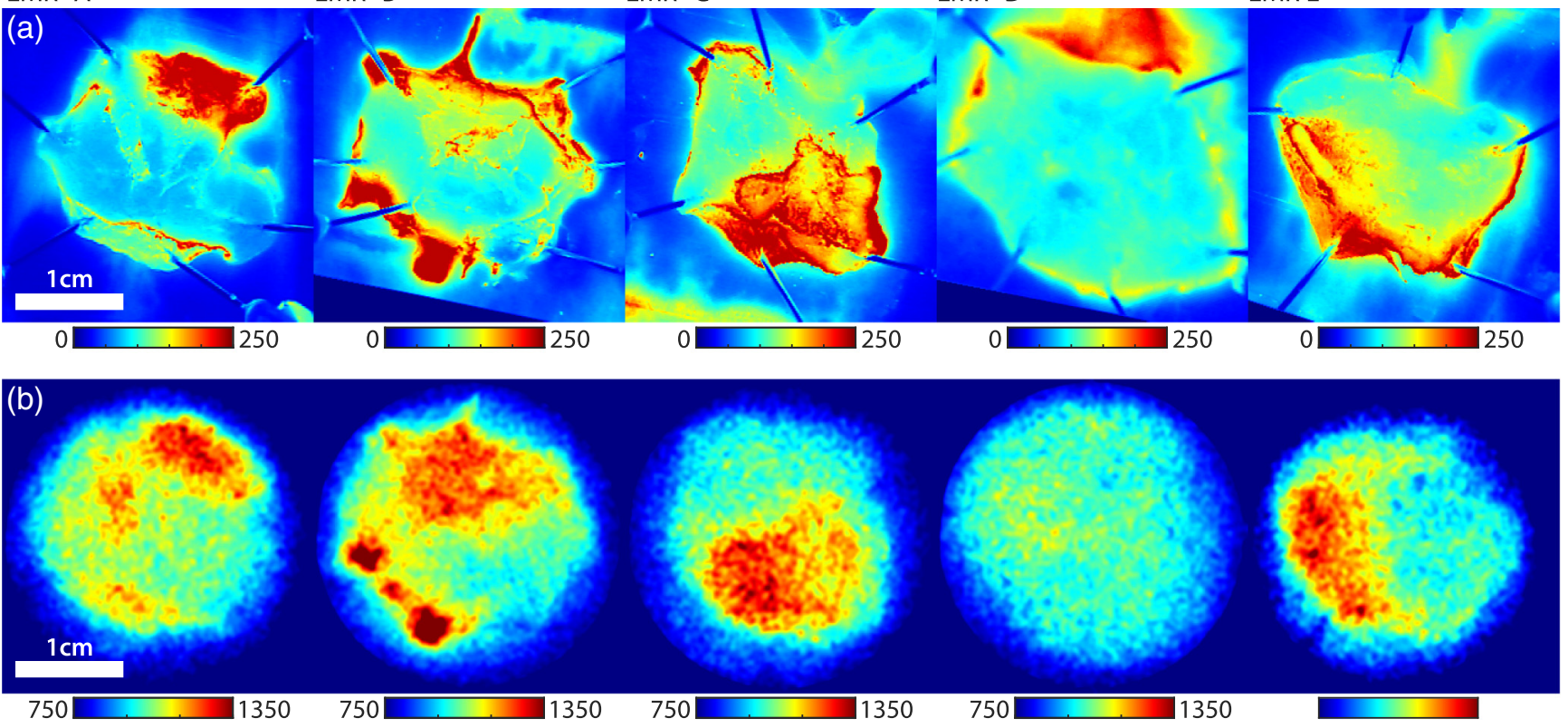

(c)
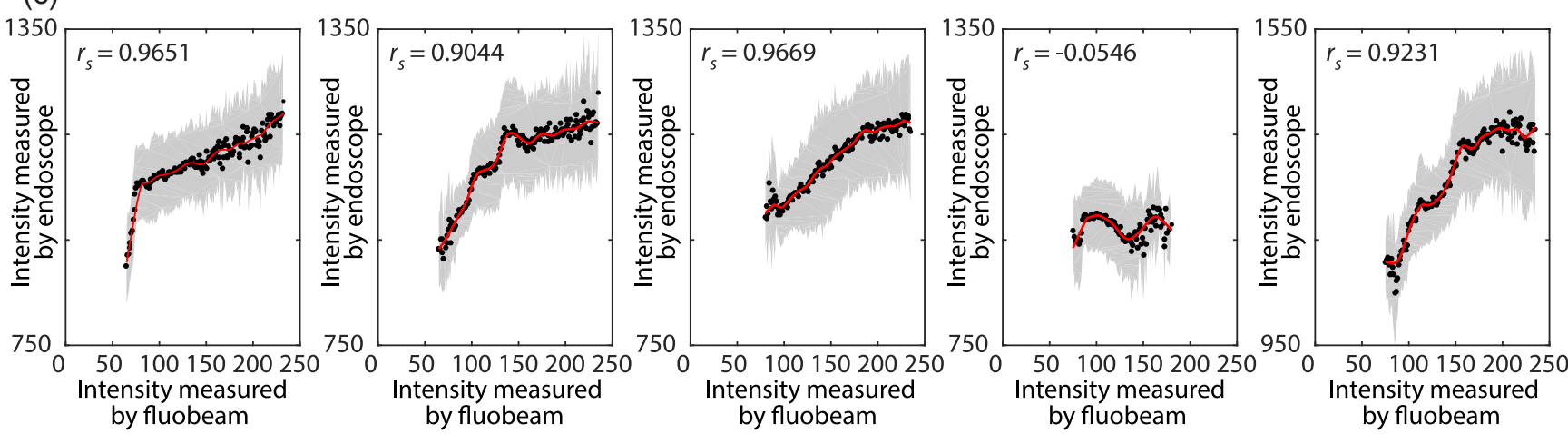

Fig. 8 Fluorescent WGA-IR800 binding to human endoscopic mucosal resection and correlation to gold standard. (a) Wide-field high resolution NIR images acquired using the Fluobeam intraoperative imaging system. (b) NIR images acquired using the bimodal endoscope. (c) Correlation between pixel intensities in the Fluobeam images and the average pixel intensities of coregistered pixels in the endoscope image. The gray areas correspond to the standard deviation of pixels in the endoscope images. A threshold was placed on the Fluobeam images in order to remove low intensity pixels (due to the pins holding tissue in place rather than signal from the tissue surface itself) and their corresponding endoscope image pixels. The threshold was determined by removing low intensity Fluobeam pixels until these pixels began to correspond to tissue as well as the pins. The same pixels were then removed from the endoscope images. The thresholds were determined to be $65,65,80,75,75$ for EMRs A to E, respectively. The red line shows a robust locally weighted regression smoothing. Spearman correlation coefficients are given within the graphs. High signal intensity observed at the periphery of the specimens is due to pooling of dye between the edge of the tissue specimen and the underlying parafilm. 
and endoscope EMR images for all collected specimens are shown in Figs. 8(a) and 8(b), respectively. The high signal observed at the edge of the specimens is due to pooling of the dye between the edge of the tissue specimen and the underlying parafilm, which remains even after washing. The intensity recorded in each coregistered pixel in the Fluobeam ${ }^{\circledR}$ and endoscope images was compared to determine if there was correlation between the images and hence whether the data acquired with our endoscope faithfully recapitulates that acquired with the gold standard Fluobeam ${ }^{\circledR}$ system.

Intensity scatter plots of these data clearly show a direct relationship between fluorescence intensity in the Fluobeam ${ }^{\circledR}$ and endoscope images for 4 of the 5 EMRs [Fig. 8(c)]. Spearman's rank correlation coefficient reveals a moderate but significant correlation between the Fluobeam ${ }^{\circledR}$ and endoscope images in these 4 of 5 EMRs $\left(r_{s}=0.90-0.97\right)$. The fluorescence signal in central $80 \%$ of the EMRs within Fluobeam ${ }^{\circledR}$ images, given as mean \pm S.D., was found to be $110 \pm 50,130 \pm 40,160 \pm 40,100 \pm 9,140 \pm 30 \mathrm{AU}$ for EMRs A to E, respectively. The lack of significant correlation in EMR D is therefore likely due to the relatively uniform fluorescence observed in both Fluobeam ${ }^{\circledR}$ and endoscope images.

Using EMR B, which had two large regions of ND and dysplastic tissue (see Sec. 2.5.2), we then coregistered the NIR endoscopy data to the histology grid [Fig. 9(a)]. The expected negative binding relationship between dysplastic and ND tissue was confirmed [Fig. 9(b)]. These results provide a preliminary indication that WGA-IR800CW fluorescence imaging with our endoscope would be capable of distinguishing between disease pathologies in esophageal tissue.

\section{Discussion}

The ability to detect areas of dysplasia in patients with Barrett's esophagus is a significant clinical challenge. The current standard of care, including WLE with random and targeted biopsies, suffers significant limitations due to the poor contrast available for dysplastic tissue in regions of Barrett's. If it were possible to accurately detect these lesions, they could be resected or ablated, preventing the progression of disease to EAC, for which prognosis is bleak with 5-year survival rates reported as low as $20 \%{ }^{1}$

To address this clinical challenge, we combined a NIR fluorescently labeled wheat germ agglutinin (WGA-IR800CW) that shows differential binding to normal, Barrett's, dysplastic, and cancerous tissue ${ }^{36}$ with a bimodal endoscope capable of acquisition and coregistration of white light reflectance and NIR fluorescence signals in real time. We show here that our system is able to detect WGA-IR800CW at concentrations as low as $110 \mathrm{nM}$ at the shortest working distances with a field of view of $63 \mathrm{deg}$ and an image resolution of $182 \mu \mathrm{m}$. When applied to image WGA-IR800CW in a realistic tissue scenario using ex vivo mouse stomachs, we were able to discriminate gastric- from squamous-type tissue. We then demonstrated, in a pilot study, the performance of the system as applied to endoscopic mucosal resection specimens from a patient with Barrett's esophagus and compared our data to a gold standard system for detecting NIR fluorescence. These results showed an encouraging correlation between fluorescence signal intensities recorded with the two systems on a per-pixel basis, relating directly to histopathological outcome. ${ }^{45}$

While these results are promising, some instrumentation challenges remain for clinical translation. In this work, we were imaging ex vivo specimens on a flat surface. Optimal (a)

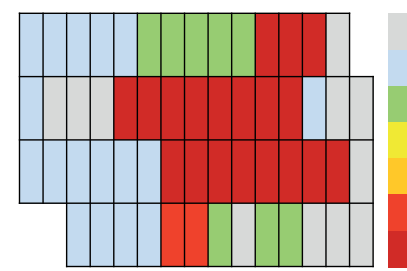

Artifact

Normal squamous

Non dysplastic barrett's Indefinite for dysplasia

Low-grade dysplasia

High-grade dysplasia

Intra mucosal carcinoma
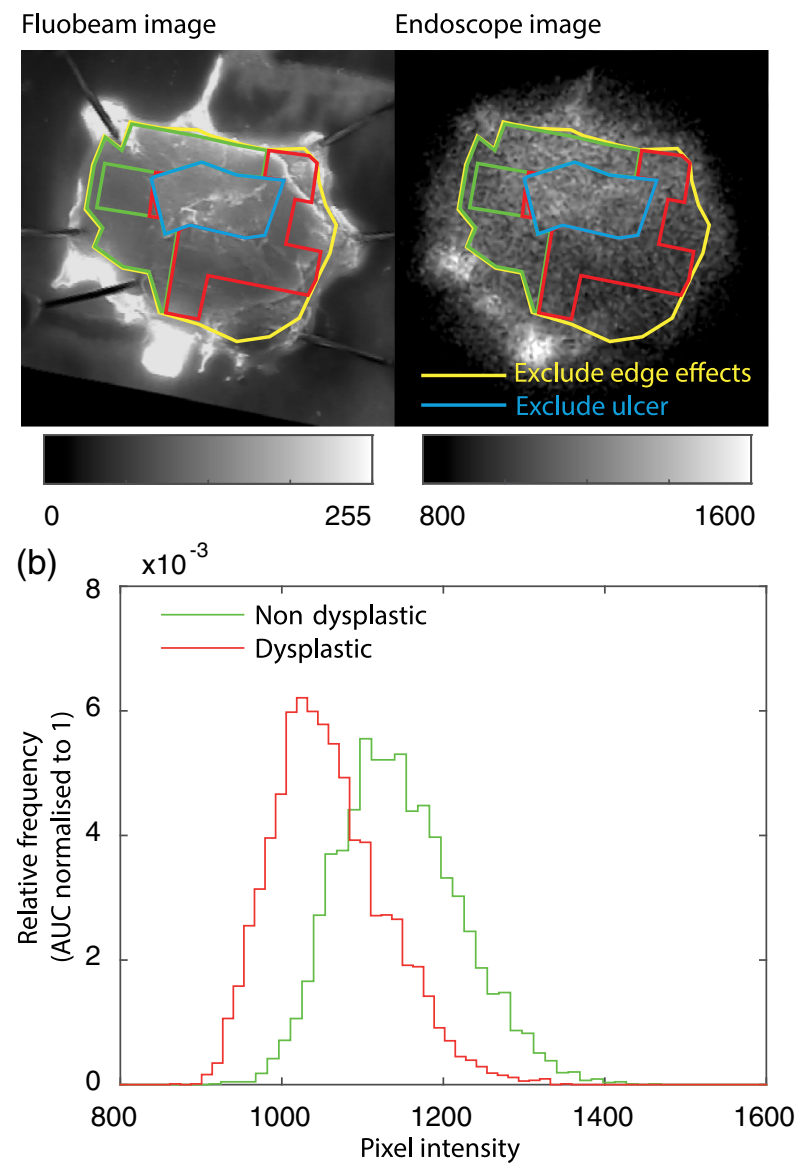

Fig. 9 Example coregistration with pathology from EMR B. (a) Histology grid manually coregistered to the Fluobeam image and then transferred to NIR endoscope image (since the Fluobeam and endoscope images were previously coregistered). The outside region was excluded to remove edge effects due to pooling of the dye between the edges of the tissue and the parafilm (yellow). An ulcer identified by the pathologist was excluded (blue). Areas labeled "artifact" in the histology grid may: contain pin holes; have no tissue present for analysis (due to the rectangular cuts); or be blurry in the scanned image. (b) To facilitate histopathological correlation, the normal oesophageal squamous epithelium and oesophageal gastric/ intestinal metaplasia (nondysplastic Barrett's) were grouped together as "nondysplastic" (ND) and any grade of neoplasia, including indefinite for dysplasia (ID), low- (LGD) and high-grade (HGD) dysplasia and intramucosal cancer (IMC), were grouped together as "dysplastic". Pixel values for the endoscope image are plotted as a histogram for the largest continuous "nondysplastic" (green) and "dysplastic" (red) regions.

sensitivity in this geometry was found for the shortest WDs in the range found in vivo as expected. Clinical endoscopy may encompass WDs up to several centimeters within a single image. If we wish to perform quantitative fluorescence imaging in vivo, we will need to correct for the variable working distance within a given image. This may be achieved by constructing a pixel-wise ratiometric image of a fluorescence image and a 
coregistered white light reflectance image, which has shown to perform well in fluorescence phantoms. ${ }^{35}$ In addition, a number of developments could be included to ensure that we achieve acceptable SNR at a wider range of WDs. We could employ a more tailored light source, such that more spectral power is deposited across the excitation band of the IRDye800CW, or increase the overall illumination power of the system given that we remain significantly below the power used in current commercial endoscopes. Improved sensitivity would also allow us to improve our FOV to approach the 140 deg FOVs that are common in commercial endoscopes.

We will also need to overcome coregistration challenges to relate our fluorescence data to the "ground truth" histopathological analysis in future studies of ex vivo specimens. Inaccuracies in the histology grid registration may arise due to: unavoidable deformations and artifacts in the processing of EMRs; and the assignment of a single majority pathological grade to a large $1 \mathrm{~mm}$ area, which may contain mixed pathologies. The manual coregistration of the grid to the fluorescence image then adds a further challenge. Application in a clinical setting in vivo also requires an optimization of the dye concentration to be used with our system. The sensitivity assays performed here used solutions of the dye in well plates. An important next step will be to calibrate how the sprayed concentration of WGA-IR800CW relates to the final bound concentration observed on the tissue following application and washing with a spray catheter. A quantitative assessment of the applied concentration of dye and specific/nonspecific binding against a known concentration of sialic acid residues will allow us determine the optimum spraying concentration for use in future clinical trials.

Our approach has several advantages over other molecular imaging methods being explored for NIR endoscopy. First, lectins are cheap, stable at low $\mathrm{pH}$, heat stable,${ }^{47}$ and have a known, validated molecular target that has been shown to be down regulated in the progression to EAC. ${ }^{36}$ Furthermore, these lectins can be topically applied with a short incubation time, minimizing the length of the procedure compared to, for example, intravenous administration of antibodies, which requires a long clearance time. Second, the use of a wide-field bimodal fluorescence endoscope allows this approach to image the entire esophagus for improved endoscopic surveillance. We therefore believe that our approach will facilitate rapid translation into future clinical trials.

\section{Conclusions}

We have developed a bimodal white light reflectance and NIR fluorescence endoscope that provides wide-field surveillance for dysplastic tissue in patients with Barrett's esophagus using the molecular imaging contrast agent WGA-IR800CW. Our endoscopy system could distinguish between squamous and gastric tissue types in mouse stomachs ex vivo and compared favorably to gold standard measurements when applied to ex vivo endoscopic mucosal resections from patients. Based on these data, we can move forward with clinical translation of this device, performing further optimization of the instrument hardware and image processing software based on our findings, prior to implementation in clinical trials.

\section{Acknowledgments}

This work was funded by the EPSRC-CRUK Cancer Imaging Centre in Cambridge and Manchester (Grant no. C197/ A16465), CRUK (C14303/A17197) and the EU FP7 agreement
FP7-PEOPLE-2013-CIG-630729. Additional funds were provided by the University of Cambridge MRC Confidence in Concept Award. SEB is supported by a CRUK Career Establishment Award (Grant no. C47594/A16267).

\section{References}

1. C. Hur et al., "Trends in esophageal adenocarcinoma incidence and mortality," Cancer 119(6), 1149-1158 (2013).

2. R. Anaparthy and P. Sharma, "Progression of Barrett oesophagus: role of endoscopic and histological predictors," Nat. Rev. Gastroenterol. Hepatol. 11(9), 525-534 (2014).

3. T. K. Desai et al., "The incidence of oesophageal adenocarcinoma in non-dysplastic Barrett's oesophagus: a meta-analysis," Gut 61(7), 970-976 (2012)

4. L. C. Duits et al., "Barrett's oesophagus patients with low-grade dysplasia can be accurately risk-stratified after histological review by an expert pathology panel," Gut 64(5), 700-706 (2015).

5. N. J. Shaheen et al., "Radiofrequency ablation in Barrett's esophagus with dysplasia," N. Engl. J. Med. 360(22), 2277-2288 (2009).

6. K. N. Phoa et al., "Multimodality endoscopic eradication for neoplastic Barrett oesophagus: results of an European multicentre study (EUROII)," Gut 65(4), 555-562 (2015).

7. R. C. Fitzgerald et al., "British Society of Gastroenterology guidelines on the diagnosis and management of Barrett's oesophagus," Gut 63(1), $7-42$ (2014).

8. D. S. Levine et al., "Safety of a systematic endoscopic biopsy protocol in patients with Barrett's esophagus," Am. J. Gastroenterol. 95(5), 1152-1157 (2000)

9. S. J. Spechler et al., "American Gastroenterological Association technical review on the management of Barrett's esophagus," Gastroenterology 140(3), e18-e52 (2011).

10. M. B. Sturm and T. D. Wang, "Emerging optical methods for surveillance of Barrett's oesophagus," Gut 64(11), 1816-1823 (2015).

11. M. I. Canto et al., "In vivo endomicroscopy improves detection of Barrett's esophagus-related neoplasia: a multicenter international randomized controlled trial (with video)," Gastrointest. Endoscopy 79(2), 211-221 (2014).

12. J. Song et al., "Meta-analysis of the effects of endoscopy with narrow band imaging in detecting dysplasia in Barrett's esophagus," Dis. Esophagus 28(6), 560-566 (2014).

13. P. Sharma et al., "Standard endoscopy with random biopsies versus narrow band imaging targeted biopsies in Barrett's oesophagus: a prospective, international, randomised controlled trial," Gut 62(1), 15-21 (2013).

14. H. C. Wolfsen et al., "Prospective, controlled tandem endoscopy study of narrow band imaging for dysplasia detection in Barrett's esophagus," Gastroenterology 135(1), 24-31 (2008).

15. C. Jayasekera et al., "Added value of narrow band imaging and confocal laser endomicroscopy in detecting Barrett's esophagus neoplasia," Endoscopy 44(12), 1089-1095 (2012).

16. M. Singh et al., "Observer agreement in the assessment of narrowband imaging system surface patterns in Barrett's esophagus: a multicenter study," Endoscopy 43(9), 745-751 (2011).

17. R. Singh et al., "Narrow-band imaging with magnification in Barrett's esophagus: validation of a simplified grading system of mucosal morphology patterns against histology," Endoscopy 40(6), 457-463 (2008).

18. D. F. Boerwinkel et al., "Third-generation autofluorescence endoscopy for the detection of early neoplasia in Barrett's esophagus: a pilot study,' Dis. Esophagus 27(3), 276-284 (2014).

19. L. M. Wong Kee Song et al., "Autofluorescence imaging," Gastrointest. Endoscopy 73(4), 647-650 (2011).

20. M. Giacchino et al., "Clinical utility and interobserver agreement of autofluorescence imaging and magnification narrow-band imaging for the evaluation of Barrett's esophagus: a prospective tandem study," Gastrointest. Endoscopy 77(5), 711-718 (2013).

21. D. F. Boerwinkel et al., "Effects of autofluorescence imaging on detection and treatment of early neoplasia in patients with Barrett's esophagus," Clin. Gastroenterol. Hepatol. 12(5), 774-781 (2014).

22. W. L. Curvers et al., "Endoscopic tri-modal imaging is more effective than standard endoscopy in identifying early-stage neoplasia in Barrett's esophagus," Gastroenterology 139(4), 1106-1114 (2010). 
23. W. L. Curvers et al., "Endoscopic trimodal imaging versus standard video endoscopy for detection of early Barrett's neoplasia: a multicenter, randomized, crossover study in general practice," Gastrointest. Endoscopy 73(2), 195-203 (2011).

24. J. D. Horwhat et al., "A randomized comparison of methylene bluedirected biopsy versus conventional four-quadrant biopsy for the detection of intestinal metaplasia and dysplasia in patients with long-segment Barrett's esophagus," Am. J. Gastroenterol. 103(3), 546-554 (2008).

25. S. Ngamruengphong, V. K. Sharma, and A. Das, "Diagnostic yield of methylene blue chromoendoscopy for detecting specialized intestinal metaplasia and dysplasia in Barrett's esophagus: a meta-analysis," Gastrointest. Endoscopy 69(6), 1021-1028 (2009).

26. S. Tholoor et al., "Acetic acid chromoendoscopy in Barrett's esophagus surveillance is superior to the standardized random biopsy protocol: results from a large cohort study (with video)," Gastrointest. Endoscopy 80(3), 417-424 (2014).

27. G. Longcroft-Wheaton et al., "Acetic acid spray is an effective tool for the endoscopic detection of neoplasia in patients with Barrett's esophagus," Clin. Gastroenterol. Hepatol. 8(10), 843-847 (2010).

28. S. H. Yun et al., "Comprehensive volumetric optical microscopy in vivo," Nat. Med. 12(12), 1429-1433 (2006).

29. H. C. Wolfsen et al., "Safety and feasibility of volumetric laser endomicroscopy in patients with Barrett's esophagus (with videos)," Gastrointest. Endoscopy 82(4), 631-640 (2015).

30. M. J. Gora et al., "Tethered capsule endomicroscopy enables less invasive imaging of gastrointestinal tract microstructure," Nat. Med. 19(2), 238-240 (2013)

31. M. L. James and S. S. Gambhir, "A molecular imaging primer: modalities, imaging agents, and applications," Physiol. Rev. 92(2), 897-965 (2012).

32. P. Habibollahi et al., "Optical imaging with a cathepsin B activated probe for the enhanced detection of esophageal adenocarcinoma by dual channel fluorescent upper GI endoscopy," Theranostics 2(2), 227-234 (2012).

33. S. Realdon et al., "In vivo molecular imaging of HER2 expression in a rat model of Barrett's esophagus adenocarcinoma," Dis. Esophagus 28(4), 394-403 (2015).

34. M. B. Sturm et al., "Targeted imaging of esophageal neoplasia with a fluorescently labeled peptide: first-in-human results," Sci. Transl. Med. 5(184), 184ra61 (2013).

35. B. P. Joshi et al., "Multimodal endoscope can quantify wide-field fluorescence detection of Barrett's neoplasia," Endoscopy 48(2), A1-A13 (2015).
36. E. L. Bird-Lieberman et al., "Molecular imaging using fluorescent lectins permits rapid endoscopic identification of dysplasia in Barrett's esophagus," Nat. Med. 18(2), 315-321 (2012).

37. C. S. von Holstein et al., "Detection of adenocarcinoma in Barrett's oesophagus by means of laser induced fluorescence," Gut 39(5), 711-716 (1996).

38. J. J. Tjalma et al., "Molecular-guided endoscopy targeting vascular endothelial growth factor a for improved colorectal polyp detection," J. Nucl. Med. 57(3), 480-485 (2016).

39. P. B. Garcia-Allende et al., "Towards clinically translatable NIR fluorescence molecular guidance for colonoscopy," Biomed. Opt. Express 5(1), 78-92 (2014).

40. R. A. Sheth et al., "Pilot clinical trial of indocyanine green fluorescenceaugmented colonoscopy in high risk patients," Gastroenterol. Res. Pract. 2016(9), 6184842 (2016).

41. J. Glatz et al., "Near-infrared fluorescence cholangiopancreatoscopy: initial clinical feasibility results," Gastrointest. Endoscopy 79(4), 664-668 (2014).

42. K. Sato et al., "Near infrared photoimmunotherapy in the treatment of pleural disseminated NSCLC: preclinical experience," Theranostics 5(7), 698-709 (2015).

43. M. Elter, S. Rupp, and C. Winter, "Physically motivated reconstruction of fiberscopic images," in Proc. Int. Conf. Pattern Recognit. 3, 599-602 (2006).

44. QImaging, "Electron-multiplying (EM) gain,” 2013, http://www. qimaging.com/resources/pdfs/emccd_technote.pdf (December 2015).

45. M. di Pietro et al., "Detection of dysplasia in Barrett's oesophagus using lectin-based near infra-red molecular imaging: an ex-vivo study on human tissue," in Proc. of the British Society of Gastroenterology Meeting (2016).

46. P. G. J. Barten, Contrast Sensitivity of the Human Eye and Its Effects on Image Quality, SPIE Press, Bellingham, Washington (1999).

47. Y. Nagata and M. M. Burger, "Wheat germ agglutinin. Isolation and crystallization," J. Biol. Chem. 247(7), 2248-2250 (1972).

Dale J. Waterhouse graduated from the University of Cambridge in 2014 with a BA MSci in natural sciences. He is currently a PhD student under the supervision of Dr. Sarah E. Bohndiek. His research is centered on the development of endoscopic imaging techniques for early detection of neoplasia.

Biographies for the other authors are not available. 